\title{
Climate Change and Behavior Patterns of Urban Residents
}

\author{
Zinaida Ivanova ${ }^{1, *}$ and Jurga Naimavičiené ${ }^{2}$ \\ ${ }^{1}$ Moscow State University of Civil Engineering, Yaroslavskoe shosse, 26, Moscow, 129337, Russia \\ ${ }^{2}$ Vilnius Gediminas Technical University, 11, Saulétekio al., 10223, Vilnius, Lithuania
}

\begin{abstract}
The aim of the study is to identify patterns of consumer's behaviour related to climate change. Research method: sociological survey. The authors conducted sociological surveys among the architecture students of the National Research Moscow State University of Civil Engineering (NRU MGSU) concerning their attitude to the climate change and the sense of personal responsibility for the current events. The results converged with the results of the European Sociological Survey (2017-2018), and discrepancies were insignificant. Drawing on the findings the authors identified behaviour models with regard for the climate change. They concluded that consumer behaviour is influenced by numerous factors, including the system of traditional values, the attitude to nature, to its resources, the government policy in respect of the climate change, as well as the effort to promote proenvironmental behaviors. Author s expanded the scope of the research and analyzed the opinions of the architecture students about the regard for climaterelated factors in architectural design. The conclusion is that the problem is not well clear to the students of the civil engineering university. Although $100 \%$ of the respondents acknowledged the need to take account of the climate-related factors in the course of urban design and construction, only $21.4 \%$ of respondents listed some elements of architecture capable of protecting the climate. The reasons for the unawareness include poor climate education, information unavailability and an overall lack of climatic culture.
\end{abstract}

\section{Introduction}

Destructive processes in the biosphere, changing familiar landscapes and eco-systems these processes have intensified over the last decade. Environmental impairment has spread over wide areas on Earth. These processes were triggered by humans, namely, human industrial activities and greenhouse gas emissions into the atmosphere that caused global warming and the climate change. Cities are among the main sources of the climate change. Urban territories make substantial contributions to this process. "Transportation, heating and cooling systems, industries and other urban activities and infrastructures act as emitters and as direct causes of climate change. Urban centers create two main categories of impacts on the carbon cycle and the climate system, namely changes related to the emission of aerosols, GHGs and solid wastes; and land-use related changes" [1].

\footnotetext{
${ }^{*}$ Corresponding author: ivanovazi@mail.ru
} 
Problems of the climate change and human behaviour are at the forefront of the science debate, as the daily living activities of humans, intensive consumption of natural resources and the way they are consumed, the attitude of societies to the exploitation of nature, and, on the whole, consumption-oriented culture pre-determine the destiny of our planet.

Consumption-oriented culture is based on the values accepted by the majority. Basic values are permanent; they are passed down from generation to generation, although things change as the time progresses. The values cherished by the groups, that an individual belongs to, produce a substantial impact on his or her behaviour pattern. Individuals behave like that because they believe that the way they behave is convenient, habitual, and comfortable, because they are sure that they behave properly. Russian ethnologist L.N. Gumilev argued that behaviour patterns are the main features of any ethnicity. The rules, followed by ethnicities, are perceived by an individual as the only behaviour pattern that he or she finds acceptable, since all other patterns look incomprehensible, unreasonable, and bizarre.

Behaviour stereotypes, typical for particular ethnicities, are manifest in different realworld situations, including consumption. Ethnic cultures also feature distinctions between the values of older and younger generations, urban residents and villagers, professional groups and social classes. Different peoples treat nature and its resources differently, and the same about their consumption models. Unlike previous epochs, contemporary European and North American cultures promote resource saving values, biosphere preservation, and swift adaptation to climate change consequences. The European Union has been developing strategic programmes aimed at the prevention of climate change processes. The mitigation of their effects and adaptation to their consequences are the two complementary approaches that reduce the risks of negative and destructive processes. Changes in the human behaviour coupled with the development of a climate saving model represent one of the principal ways of mitigating and decelerating the climate change.

\section{Literature review Research}

Climate change problems have become the subject of the most animated discussion held across the world, although the degree of involvement in the research and debate over climate-related problems varies significantly from country to country. The largest number of works is published in the countries of Western Europe and North America. Australia, New Zealand, China and Japan are being actively invited to join the research process. Publications cover various aspects of climate change. The largest number of articles is focused on the impact produced by the climate change on human health and well-being. For example, the article, written by E. Affuzo, a US-based researcher, assesses the potential impact of the climate change on the well-being of consumers in Greenland, the country whose economy is dominated by its geographical position, as it is located in the Arctic region, and it is heavily impacted by its climate and strongly dependent on it. Presently, this impact has aggravated, and the ice mass loss may influence the demand for disposable commodities and reduce the well-being of consumers in Greenland [2]. How sensitive are people to the climate change and its consequences? Do people realize the potential impact that will be produced by climate-related processes on their welfare? Do they trust the conclusions made by the researchers and the warnings made by the climatologists? These issues have been extensively covered in numerous research articles [3 - 6]. The article entitled "The role of trust for climate change mitigation and adaptation behaviour: A metaanalysis" is among these works. The co-authors have studied the role of confidence in government authorities, researchers, ecological groups and society as a whole in respect of various patterns of ecological behaviour. The co-authors make recommendations to climatologists and experts in respect of confidence building techniques and promotion of 
ecological behaviour patterns [7]. Some researchers study features of the human behaviour in relation to the climate change that are manifest in different countries and across versatile social groups. Do people feel responsible for their actions? Are they ready to change their behaviour patterns in terms of consumption of water, energy, and natural resources? What external measures are needed to change consumer behaviour patterns? $[8,9]$ The behaviour of various population groups in relation to the climate change has not been classified yet; however, it can be compared with a classification of ecological behaviour. Several research projects focus on the analysis of different types of ecological behaviour. The article, written by Spanish researchers, analyzes the relationship between objective and subjective knowledge and ecological behaviour, and the interrelation between behaviour patterns, on the one hand, and socio-economic and personal features of consumers, on the other hand. The conclusions, made by the co-authors, are backed by the findings of a representative cross-national survey conducted among Spanish citizens, aged 18 to 90 [10]. A group of South Korean and North American researchers identified three types of ecological behaviour (green purchase behavior, good citizenship behavior, and environmental activist behavior) and studied their drivers and interrelationship. The poll also tested a model in which the value orientation, perceived consumer efficiency/effectiveness (PCE) and environmental concern have an influence on the three types of behavior [11]. We can also emphasize the publications showing the influence of particular factors on the formation of specific types of climate-related behaviour [9]. For example, the article, written by the Australian researchers, shows the influence of decisions to buy personal cars on climaterelated behaviour [12].

\section{Research Tasks and Methods}

The goal of this research is to identify behaviour models of urban residents, developed under the influence of energy efficiency and climate change: do urban residents acknowledge the existence of climate change? Do they feel responsible for it and what do they do to mitigate its consequences? Behaviour patterns are influenced by various factors. First of all, they are influenced by the group affiliation and a system of values typical for the group. The research objective is to identify these factors and the way they are manifest through practical activities.

Methods of research:

1) The analysis of documents. The scope of documents, thus analyzed, encompasses laws and government resolutions, decrees issued by local government authorities and focused on climate saving and adaptation of cities and territories to the climate change. Besides, the author has analyzed the findings of the European Social Survey (ESS) in 2017 ("European Attitudes to Climate Change and Energy: Topline Results from Round 8 of the European Social Survey") [13].

2) The sociological survey, or the questionnaire survey. Two questionnaire surveys were conducted, the topic of the first one reads as "What do urban residents do to mitigate the adverse consequences of the climate change?". The second survey was conducted among the students majoring in architecture and its topic reads as "In what way should architecture take account of the climate change?" The survey was conducted among young people, students of the National Research Moscow State University of Civil Engineering (NRU MGSU), majoring in architecture and residing in Moscow and the Moscow Region (Russia). The survey was conducted in April and May, 2020. 


\section{Research results}

The survey has proven that urban and rural residents have just initiated the development of more or less conscious behaviour in respect of the climate change. Although the consequences of the climate change are well-known, and they have caused irreversible changes in some areas on our planet, public consciousness has not had enough time to convert this fact into personal psyche and convictions. Sociological surveys, conducted in different countries, have shown that the fact of the climate change has been acknowledged somewhere at the level of depthless consciousness. According to the respondents, they are aware of the climate change and they realize that humans are probably responsible for it; however, the comprehension and understanding of the personal and public responsibility for this phenomenon is a slow process.

\section{Findings of the European Social Survey (ESS)}

In 2017, ESS conducted Round 8 of the sociological survey (interviews) in 23 countries of Europe. Over 44 thousand respondents participated in the poll [13]. The questions were to assess the mental image of the climate change, in particular, the opinions concerning the existence of the climate change, its origin and consequences.

The question was asked of the respondents whether they believed that the climate was likely to change or it was definitely changing. Over $90 \%$ of the respondents in the majority of the European countries responded that the climate was likely to change. Iceland ranks number one among the respondents who share this opinion. In Iceland, $97.7 \%$ of the respondents think that the climate is changing. Perhaps, this viewpoint is popular because the consequences of the climate change are highly pronounced in Iceland. For example, in 2014, Okjökull glacier almost disappeared, although it was almost 700 years old. Icelanders commemorated the glacier with a plaque. The smallest number of respondents who believe that the climate is changing, lives in Israel (86\%) and Russia (82\%). Probably, the climate change is not pronounced in these countries. The next question, asked of the respondents, read as follows: do they believe that the climate change is to some extent caused by human activities? Different nations answered this question differently: over 94\% of German residents and Icelanders believe that it's true; residents of Israel, Norway and five countries of Eastern Europe are less convinced that the climate change is, to some extent, triggered by human activities.

Respondents' concerns about the climate change are relatively insignificant across Europe. Climate change fears are particularly strong in Portugal, Spain, and Germany, provided that over $50 \%$ of the Portuguese population express deep concerns about this problem. In fact, climate change concerns are relatively low in East European countries, such as Lithuania, Estonia, Poland, and Russia, less than $20 \%$ of their population are concerned with climate change problems (Fig. 1). 


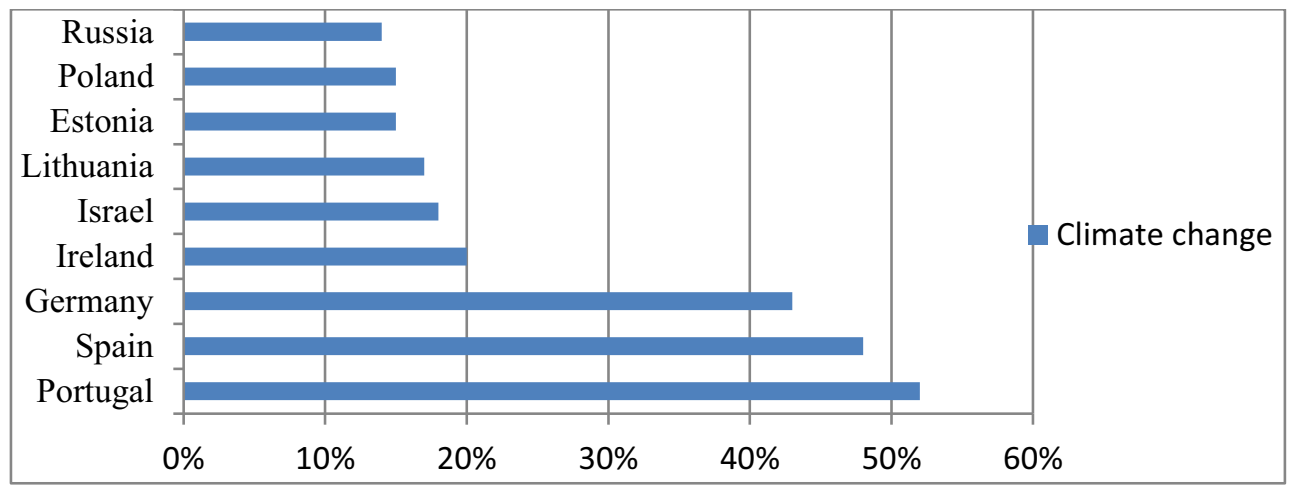

Fig. 1. Climate change concerns [13].

The individuals whose behaviour served as the source material for the behaviour patterns, could have assumed personal responsibility for current developments in order to overcome the climate change consequences. Within the framework of Round 8 of the European Social Survey, questions were asked about the sense of personal responsibility and individual contributions to the mitigation of climate change consequences. A scale of eleven, where zero meant "not at all" and ten meant "a lot" was offered to the respondents. The sense of personal responsibility was the highest in France (personal norms), Switzerland, Germany (where it scored seven), and the lowest in the Czech Republic and Russia (Fig. 2). An interrelation was identified: the respondents, who do not believe in the climate change, do not feel personally responsible for it and efficient in mitigating it.

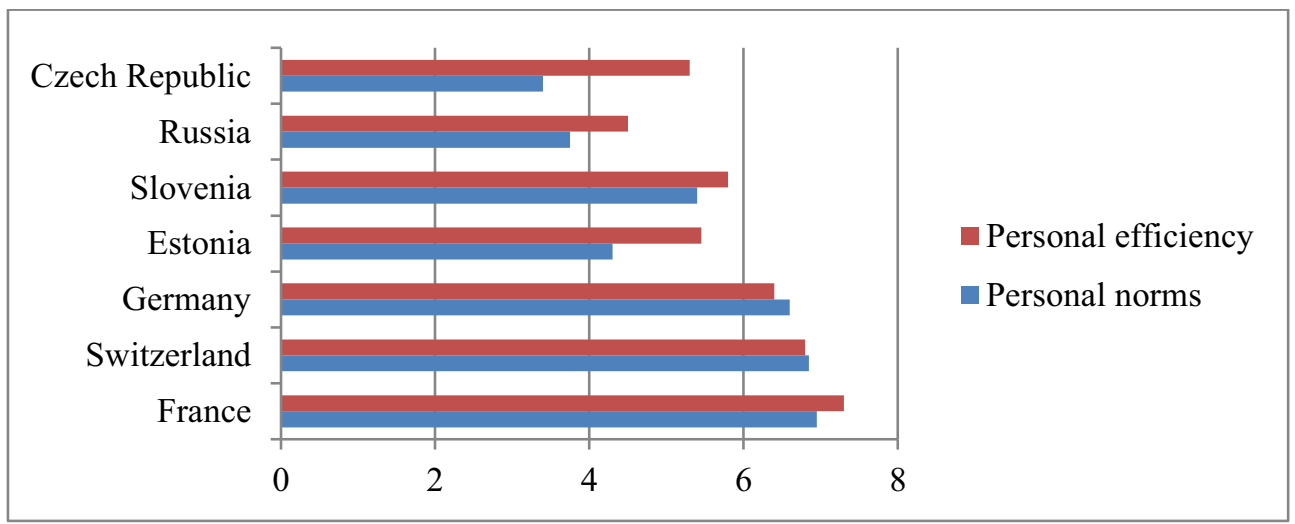

Fig. 2. Personal norms and personal efficiency [13].

Numerous respondents, mainly those based in Hungary, the Czech Republic, and Russia, are not sure that they could take any action to mitigate the climate change; neither are they sure that their power consumption, if limited, is likely to decelerate the climate change process.

\section{Findings of the sociological surveys conducted at the Moscow State University of Civil Engineering (NRU MGSU)}

Two sociological surveys were conducted among the bachelor students majoring in architecture. We assume that architects are responsible for the aggravation of climaterelated problems, since they design buildings that emit greenhouse gases and contribute to ecological degradation of cities. The first survey was conducted among 56 students, including $28.6 \%$ males and 71.4 females. $75 \%$ of the respondents reside in Moscow; others 
live in the towns in the Moscow Region. To begin with, we asked a question whether the students were aware of the climate change problem. 52 out of 56 respondents (or 92.9\%) had pondered about the climate change and its consequences; however, only $78.6 \%$ of the respondents believed that this problem was relevant. $19.6 \%$ of the respondents were convinced that this problem was exaggerated and it did not deserve any deliberate attention. As for the question about the respondents' awareness of the adverse consequences of the climate change, only $19.6 \%$ of the respondents were sure that they had enough knowledge about the adverse consequences of the climate change, $78.6 \%$ knew little about it, $5.4 \%$ of the respondents knew nothing about the problem. However the majority of the students responded that there was insufficient climate change information available in Russia. As for the question about the intensity of the influence produced by the climate change on human lives, $32 \%$ of the respondents insisted that the climate change made a strong impact, and that they could feel it themselves (average annual temperatures rise, the air is polluted); $50 \%$ considered the impact insignificant. $18 \%$ of the respondents believed that climate change produced no impact at all.

The questionnaire also had a question whether the respondents felt personally responsible for the climate change, and $73 \%$ of the students gave a positive answer, while others answered in the negative. As for the question about the efficiency of personal behaviour in respect of climate change deceleration, $50 \%$ of the respondents answered that they were taking particular actions themselves; $42 \%$ took no actions, and $12.5 \%$ had no intention to take any action.

Are young people ready to take any action to mitigate the climate change consequences? The respondents were to answer the question whether they were ready to give up their motor vehicles and travel by bike. $44.6 \%$ of the respondents were enthusiastic about the idea and answered that they were ready to travel by bike or scooter; $46.4 \%$ thought it was acceptable if the weather was fine; $9 \%$ had no intention to use a bike. When analyzing the answers to this question, one should bear in mind the fact that the respondents were 20-21 years old, and they were university students, therefore, they treated cycling as fun. As for the question about their willingness to pay more for their electric vehicle journeys, $62.5 \%$ were ready to do it, while $39.3 \%$ were not.

Another question dealt with their personal efficiency in terms of the climate action, including the reduction of the energy consumption. The question read as follows: "Do you unplug electric appliances, when they are not used?" $60.7 \%$ of the respondents answered in the negative, while $39.3 \%$ answered in the affirmative. Students also made numerous suggestions concerning changes in their personal behaviour: waste sorting, giving up using plastic bags, giving up waste combustion, expanding green areas covered with plants, etc.

When answering a set of questions dealing with the government's fight the against the climate change, some students were in doubt whether everything depended on the state. One third of the respondents $(30.4 \%)$ thought that every citizen was responsible for this problem, others were sure that only government authorities could organize and hold climate actions and perform law enforcement functions. Students recommended the government to change the climate policy and to ensure strict control over the following climate change boosters:

1) Reduce crude oil/gas recovery expenses and switch to renewable sources of energy (76.8\%). Indeed, $64 \%$ of the respondents are ready to pay higher tax rates, if renewable sources need bigger funding;

2) Ban diesel-fueled vehicles (58\%);

3) Impose restrictions on airflights $(24.1 \%)$;

4) Impose restrictions of launches of spacecrafts (21.4).

Therefore, evidently, on the whole, the findings of our survey correlate with those of the survey launched by the European Social Survey; however our project has identified a larger 
number of young people unwilling to engage in civic activities. It comes as a surprise, as these young respondents are future architects, designers, and urban planners. They will assume responsibility for numerous projects, that will entail ecological urban environments, designs of sustainable buildings and structures, and application of ecological construction materials.

The topic of the second survey reads as "In what way should architecture take account of the climate change?"

44 NRU MGSU students, majoring in architecture, participated in the survey. $95 \%$ of the respondents acknowledged that contemporary items of architecture produce an adverse impact on the climate, as they are made of hazardous construction materials. $100 \%$ of the respondents believe that climate should be taken account of in the process of architectural design. However, when the respondents were to answer the question "What climate protection elements should be integrated into an architectural design project?", the majority of the students $(78.6 \%)$ gave no answer, $21.4 \%$ listed such elements as green roofs and walls, "protective" facades and coatings, nanofacades, aerodynamic shielding, solar shading, ecological materials, technologies similar to muxarabi, solar and wind power use (see Fig. 3).

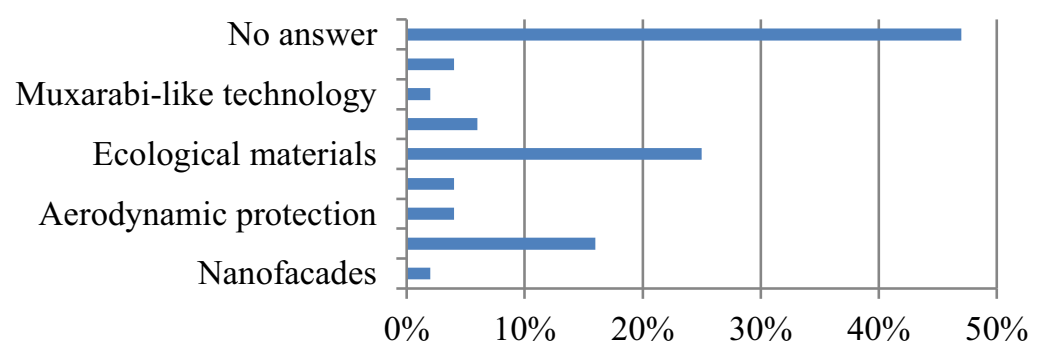

Fig. 3. A breakdown of the answers to the question "What climate protection elements should be integrated into an architectural design project?"

The same respondents, who have learned the principles of climate protection architecture very well, use climate protection elements in their architectural projects (Fig. 4).

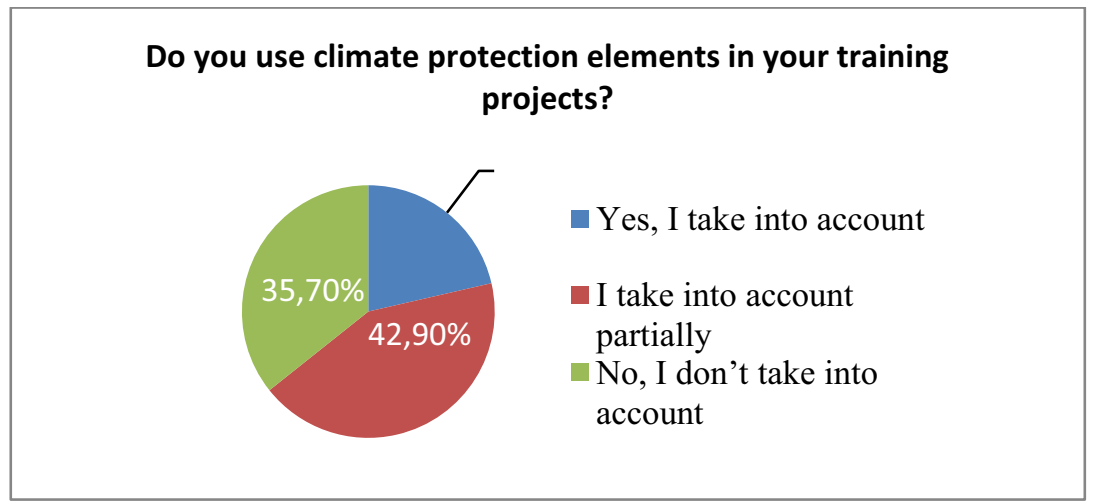

Fig. 4. A breakdown of answers to the question "Do you use climate protection elements in your training projects?" 
Unfortunately, on the whole, students are unaware of the principles of sustainable development and sustainable architecture. Over $50 \%$ of the respondents gave no answer to this question.

\section{Discussion}

The simulation of human behaviour is a kind of prognostication of human actions and a forecast of their conduct in particular contexts, the choices that they are going to make, and the outcomes that government authorities will deal with in the future [14]. This behaviourfocused survey has proven the need for an in-depth understanding of the motives and the logic of the human behaviour. People do not always act rationally. Their behaviour is influenced by numerous factors, primarily, by the values and norms, typical for the groups that they belong to [15]. Having mastered the logic of the human behaviour and studied the factors that trigger particular choices, one can identify behaviour patterns, and classify them in order to develop a policy focused on the problem [16]. Having developed the behaviour patterns that simulate the actions of individuals and social groups performed in response to climate changes, one can get to understand further actions of people, or what they are ready to do and what they are not going to do whatever happens. The degree of human sensitivity to emerging circumstances is another factor to be studied, as well as the extent of responsiveness of individuals and social groups to adverse consequences of the climate change, and how individuals and organizations perceive risks or uncertainties, whether they are capable of taking account of these risks and suggesting their responses. If this information is available, one can develop a state, region-, city- and village-wide climate policy. Methods of assessment, as well as sociological, psychological and ethical techniques will assist researchers in their decision-making process.

Climate policy planning and implementation encompasses the mitigation of adverse climate change consequences. Climate policy adaptation at each level depends on social values, goals and patterns of risk perception by the social groups and individuals [15]. The acknowledgement of versatile concerns, circumstances, sociocultural contexts and anticipations will facilitate decision-making processes. Knowledge systems, typical for versatile peoples, ethnic and traditional practices applicable to the environment, can serve as the source material used to develop the framework adaptation patterns when dealing with the climate change and its consequences. The integration of these types of knowledge into current practices improves the efficiency of the climate change mitigation and adaptation efforts.

\section{Conclusions}

1. Behaviour patterns, encompassing features of traditional values, ethics of economic relationships, lifestyles of different peoples, were developed on the basis of the analysis of various documents and findings of the European Social Survey (ESS). The government policy, implemented in the climate change legislation, decisions dealing with the national economy and taxation system, encouragement/punishment of individuals for their actions, are the most important factors of influence on behaviour patterns. Awareness building, upbringing and education, namely, promotion of energy efficient behaviour and its advantages represent the second factor.

2. The behaviour patterns thus generated include:

1) Behaviour pattern typical for Scandinavian peoples;

2) Behaviour pattern typical for peoples of Central Europe;

3) Behaviour pattern typical for peoples of Southern Europe; 
4) Behaviour pattern typical for peoples of Eastern Europe and Baltic states;

5) Behaviour pattern typical for Russia.

The countries located in one and the same region feature similar perception models and concerns, let alone similar feedbacks. For example, concerns about electricity access are rather high in Spain (21\%) and Portugal (22\%). Access to power supply also worries Italians $(70 \%)$ and Portuguese (6\%) [13]. The studies show that people concerned with the accessibility of energy, take fewer actions; they neither support the government energy policy, nor believe in any future changes in power consumption patterns. This finding questions (1) the adequacy of methods, used to build the public awareness about the energy accessibility and (2) the due regard for such behaviour in the process of government policy development [17].

3. The behaviour pattern typical for Russians arouses concerns. Pursuant to the survey findings, the awareness about climate change processes is very low even among the social groups capable of influencing climate change processes (here we speak about architects). Therefore, the survey has identified a large percent of people who are indifferent to climate problems and do not take any action. It is not true that Russians are indifferent to nature. Since ancient times, Russian people have honoured nature and cared for the earth. However Russians tend to behave in the bizarre manner, they have been demonstrating utterly inconsistent behaviour patterns, and, on the whole, they are not focused on saving resources. Russian people are wasteful; they do not save water, energy or natural resources. The authorities behave in the same way. Russia pursues neither any active, nor any efficient climate policy; no effective actions are taken, and the awareness building effort is insufficient. The Russian climate doctrine, approved in 2009, acknowledges the fact of global warming and the validity of the hypothesis about its anthropogenic origin. Russia ratified the Paris climate agreement, and the country argues that the climate change is a threat to the ecological balance, that it aggravates the risks that may threaten the key branches of the national economy, human health and lives. In 2019, the development of the National implementation plan attached to the Paris climate agreement was completed. However, this document entails no specific actions. It look like the passive position of the federal authorities, the lack of any specific ideas concerning further actions, the failure of any awareness building effort, little attention to the climate change at high schools and universities contribute to the generation of passive behaviour patterns demonstrated by the Russian respondents (within the framework of the survey conducted by the European Social Survey) and the architecture majors who participated in the survey conducted at NRU MGSU.

This research was carried out under the BECK (598746-EPP-1-2018-LT-EPPKA2CBHE-JP "Integrating education with consumer behaviour relevant to energy efficiency and climate change at the Universities of Russia, Sri Lanka and Bangladesh") project funded with support from the European Commission. The findings and opinions reported in this paper reflect the views only of the authors, and the Commission cannot be held responsible for any use which may be made of the information contained in it.

\section{References}

1. Cities and Climate Change: Policy Directions Global Report on Human Settlements 2011. United Nations Human Settlements Programme. https://unhabitat.org/globalreport-on-human-settlements-2011-cities-and-climate-change

2. E. Affuso. Consumer welfare and climate change in Greenland. Energy Economics, 84, 104505 (2019) https://doi.org/10.1016/j.eneco.2019.104505 
3. A. Musacchio, L. Andrade, E. O'Neill et al. Planning for the health impacts of climate change: Flooding, private groundwater contamination and waterborne infection - A cross-sectional study of risk perception, experience and behaviours in the Republic of Ireland. Environmental Research, 194, $110707 \quad$ (2021) https://doi.org/10.1016/j.envres.2021.110707

4. F. Knobloch, M.A.J. Huijbregts, J.-F. Mercure. Modelling the effectiveness of climate policies: How important is loss aversion by consumers? Renewable and Sustainable Energy Reviews, 116, 109419 (2019) https://doi.org/10.1016/j.rser.2019.109419

5. F. Rizzi, E. Annunziata, M. Contini, M. Frey. On the effect of exposure to information and self-benefit appeals on consumer's intention to perform pro-environmental behaviours: A focus on energy conservation behaviours. Journal of Cleaner Production, 270, 122039 (2020) https://doi.org/10.1016/j.jclepro.2020.122039

6. T.M. Lee, E.M. Markovitz, P.D. Howe, et al. Predictors of public climate change awareness and risk perception around the world. Nature Climate Change 5, 1014-1020 (2015). DOI:10.1038/nclimate2728

7. Cologna V., Siegrist M. The role of trust for climate change mitigation and adaptation behaviour: A meta-analysis. Journal of Environmental Psychology, 69, 101428 (2020) https://doi.org/10.1016/j.jenvp.2020.101428

8. J. Batalla-Bejerano, E. Trujillo-Baute, M. Villa-Arrieta. Smart meters and consumer behaviour: Insights from the empirical literature. Energy Policy, 144, 111610 (2020) https://doi.org/10.1016/j.enpol.2020.111610

9. T.-K. Yu, F.-Y. Lin, K.-Y. Kao, C.-M. Chao, T.-Y. Yu. An innovative environmental citizen behavior model: Recycling intention as climate change mitigation strategies. Journal of Environmental Management? 247, 499-508 (2019) https://doi.org/10.1016/j.jenvman.2019.06.101

10. L.V. Casaló, J.-J. Escario, C. Rodriguez-Sanchez. Analyzing differences between different types of pro-environmental behaviors: Do attitude intensity and type of knowledge matter? Resources, Conservation and Recycling, 149, 56-64 (2019) https://doi.org/10.1016/j.resconrec.2019.05.024

11. Y.-K. Lee, S. Kim, M.-S. Kim, J.-G. Choi. Antecedents and interrelationships of three types of pro-environmental behavior. Journal of Business Research, 67 (10), 2092105 (2014). https://doi.org/10.1016/j.jbusres.2014.04.018

12. M.A. Saleem, L. Eagle, D. Low. Climate change behaviors related to purchase and use of personal cars: Development and validation of eco-socially conscious consumer behavior scale. Transportation Research Part D: Transport and Environment, 59, 68-85 (2018). https://doi.org/10.1016/j.trd.2017.12.023

13. W. Poortinga, G. Böhm, L. Steg, et al. 2018. European Attitudes to Climate Change and Energy: Topline Results from Round 8 of the European Social Survey. Published by the European Social Survey ERIC City, University of London Northampton Square, London EC1V OHB, United Kingdom. http://www.europeansocialsurvey.org/docs/findings/ESS8_toplines_issue_9_climatecha ng.pdf

14. M. Singh, M. Duggirala, H.C. Hayatnagarkar et al. Towards Fine Grained Human Behavior Simulation Models. 2016 Winter Simulation Conference (WSC). Washington, DC, USA. DOI: 10.1109/WSC.2016.7822375

15. J.I.M. De Groot, L. Steg. Relationships between value orientations, self-determined motivational types and pro-environmental behavioural intentions. Journal of 
Environmental Psychology, 30 30

(4), $\quad 368-378$

(2010)

https://doi.org/10.1016/j.jenvp.2010.04.002

16. L. Niamir, O. Ivanova, T. Filatova. Economy-wide impacts of behavioral climate change mitigation: Linking agent-based and computable general equilibrium models. Environmental Modelling and Software. 134, 104839 (2020) https://doi.org/10.1016/j.envsoft.2020.104839

17. P. Wicker, S.Becken. Conscientious vs. ambivalent consumers: Do concerns about energy availability and climate change influence consumer behaviour? Ecological Economics, 88, 41-48 (2013) https://doi.org/10.1016/j.ecolecon.2013.01.005 